\title{
Graphene-based Materials in Gas Sensor Applications: A Review
}

\author{
Siti Zulaikha Ngah Demon, ${ }^{1}$ Ainnur Izzati Kamisan, ${ }^{2}$ Norli Abdullah, ${ }^{1}$ \\ Siti Aminah Mohd Noor, ${ }^{2}$ Ong Keat Khim, ${ }^{2}$ Noor Azilah Mohd Kasim, ${ }^{2}$ \\ Muhd Zu Azhan Yahya, ${ }^{3}$ Nor Azlian Abdul Manaf, ${ }^{1}$ \\ Ahmad Farid Mohd Azmi, ${ }^{1}$ and Norhana Abdul Halim ${ }^{2 *}$ \\ ${ }^{1}$ Centre for Defence Foundation Studies, Universiti Pertahanan Nasional Malaysia, Kem Sungai Besi, \\ 57000 Kuala Lumpur, Malaysia \\ ${ }^{2}$ Research Centre for Chemical Defence, Universiti Pertahanan Nasional Malaysia, Kem Sungai Besi, \\ 57000 Kuala Lumpur, Malaysia \\ ${ }^{3}$ Faculty for Defence Science and Technology, Universiti Pertahanan Nasional Malaysia, Kem Sungai Besi, \\ 57000 Kuala Lumpur, Malaysia
}

(Received July 11, 2019; accepted January 6, 2020)

Keywords: graphene, graphene oxide, gas sensor, graphene synthesis, electrochemical, nanocomposites

Graphene and chemically modified graphene can be fabricated via numerous routes each with its own merits concerning ease of processability, cost-effectiveness for large-scale production, and also health and safety. One of the promising applications of graphene-based composites is gas sensing, which is mainly useful for environmental monitoring. We review some of the significant findings on graphene-based sensing materials for the detection of organic vapors, toxic gases, and chemical warfare agent simulants using an electrochemical method. Electrochemical sensing can be performed by inducing interactions between gas molecules and a graphene layer, such as charge transfer that gives a change in an electrical signal. The intrinsic properties of graphene and its role in some gas sensing applications will be discussed. Graphene and graphene oxide (GO) work as continuous conductive networks with a large number of surface adsorption sites for many gas molecules. Hybrid graphene devices incorporate semiconductors, metals, and molecular binders to enhance the capabilities of solidstate gas sensors. This article also addresses current approaches to the commercialization of graphene-based gas sensors.

\section{Introduction}

A sensor is an analytical device that consists of an active sensing material with a signal transducer. It detects changes in its environment and sends the information (signal) to other electronic devices for data acquisition and interpretation. In every aspect of industrial and technological development to improve the quality of life on earth, the utilization of sensors has become crucial. ${ }^{(1-3)}$ Gas sensors specifically have been used to monitor the quality of air and the environment, and to detect toxic gases. ${ }^{(4,5)}$ The first commercially available gas sensor

*Corresponding author: e-mail: norhana@upnm.edu.my

https://doi.org/10.18494/SAM.2020.2492 
based on a platinum wire was introduced in 1923. It was Naoyoshi Taguchi who patented the first metal oxide gas sensor that later became the most commonly used gas sensor. ${ }^{(6)}$ His investigation was triggered by a propane gas explosion at Lake Yamanaka, and the first Taguchi gas sensor (TGS) was made from a tin oxide $\left(\mathrm{SnO}_{2}\right)$ film. Metal-oxide-based gas sensors are explored because of their wide semiconductor tunability and morphology architectures that exhibit a high sensitivity, low operating temperature, short response time, and high thermal stability. ${ }^{(4)}$ A variety of semiconductor metal oxide nanomaterials with hierarchical structures have been successfully prepared using the solution-phase chemical method, which is convenient for the large-scale production of various compounds, such as zinc oxide ( $\mathrm{ZnO})$, titanium oxide $\left(\mathrm{TiO}_{2}\right)$, iron oxide $\left(\mathrm{Fe}_{2} \mathrm{O}_{3}\right)$, and tungsten oxide $\left(\mathrm{WO}_{3}\right){ }^{(4,6-8)}$ Recently, carbonbased materials, such as graphene and carbon nanotubes, have been explored as gas sensing materials. ${ }^{(4,9)}$ Sensing materials have important roles in the molecular-level detection and distinction of contaminants in environmental processes. ${ }^{(10)}$ The need to understand the underlying mechanism becomes paramount for the development of sensing materials with focus on the sensor's response speed, selectivity, sensitivity, and stability. Graphene-based materials, including reduced graphene oxide (GO)-based materials, have been cited by many to be promising materials for gas sensing applications, and their rising popularity over the years is illustrated in Fig. 1. ${ }^{(1)}$

Graphene was discovered by Andre Geim and Konstantin Novoselov in 2004. ${ }^{(12,13)}$ Its discovery led to the Nobel Prize in Physics in 2010. Graphene, mainly known for its high electrical conductivity, large surface area $\left(2630 \mathrm{~m}^{2} \mathrm{~g}^{-1}\right)$, and high charge carrier mobility $\left(15000 \mathrm{~cm}^{2} \mathrm{~V}^{-1} \mathrm{~s}^{-1}\right)$ at room temperature, is attractive for various electronic applications. ${ }^{(14-16)}$ Two-dimensional (2D) carbon exists as an isolated layer from a stack of carbon sheets in graphite. Because of this, it can be considered as the basic structure of carbon materials. Figure

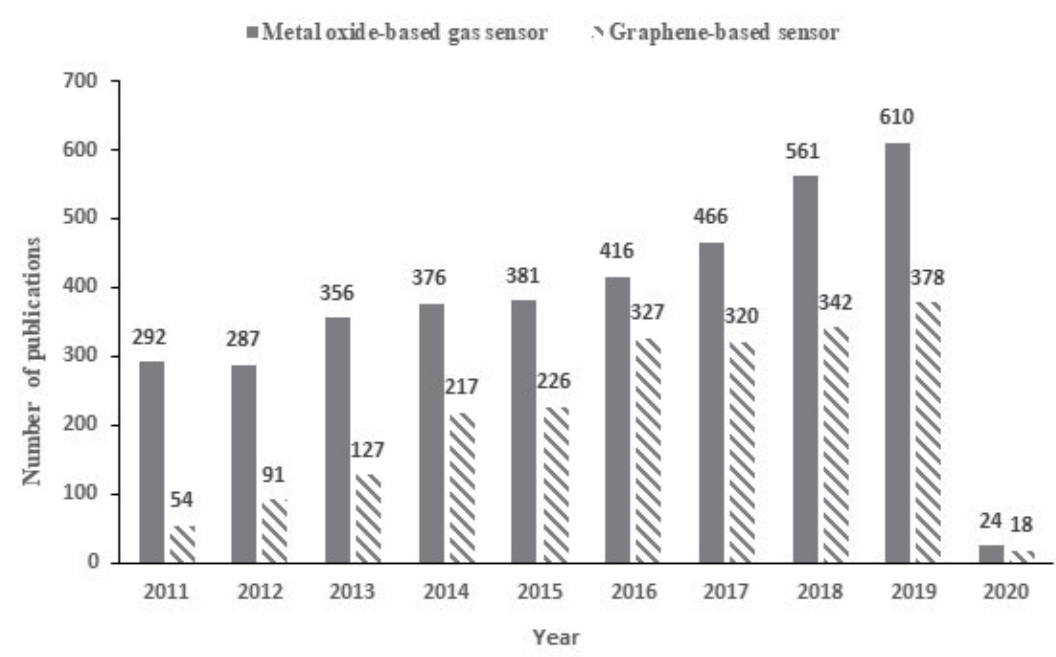

Fig. 1. Number of papers published in the areas of metal-oxide- and graphene-based gas sensors from 2011 to 2020. Data were obtained from Scopus on Dec. 12, 2019. Keywords for search: graphene, metal oxide, gas, and sensor. 


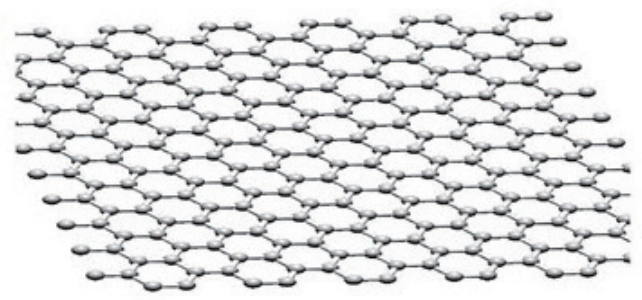

(a)

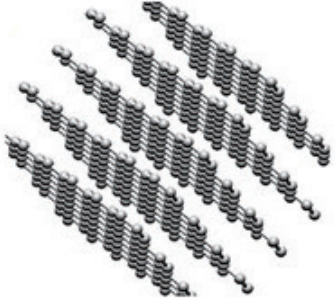

(b)

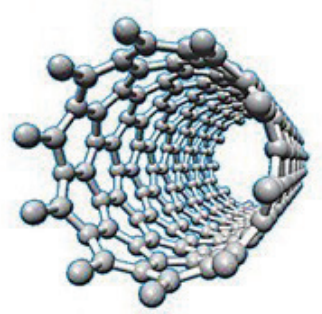

(c)

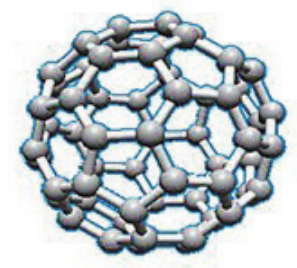

(d)

Fig. 2. (Color online) (a) Graphene as the basic structure of other graphitic materials can be (b) stacked up to form graphite, (c) rolled to form nanotubes, and (d) wrapped up to form a buckminster fullerene.

2 shows two other famous carbon-based structures, namely, carbon nanotubes (discovered by S. Iijima in 1991) and a fullerene (discovered by H. W. Kroto in 1985). The increasing research on graphene indicates that its full potential has not been achieved since its discovery. Previously, carbon nanotubes were reported to be an excellent material for solid-state gas sensors, but over the last two decades, graphene has emerged to be a more advantageous material in this particular field. ${ }^{(15)}$ It has been suggested that graphene is capable of absorbing a large amount of hydrogen, implying that it is a better gas-adsorbing material. ${ }^{(15)}$ Contrary to metal oxide sensors, graphene demonstrated low noise sensing ability at low temperatures. ${ }^{(17,18)}$ The aim of this review is to discuss several significant findings and roles of graphene in gas sensor applications. The paper will be divided into three parts: (i) classes of gas sensors, (ii) synthesis of graphene, and (ii) application of graphene in electrochemical gas sensors.

\section{Classes of Gas Sensors}

Gas sensor performance can be described by several parameters including sensitivity, selectivity, detection limit, response time, and also response recovery. Sensor sensitivity can be expressed in terms of $\mathrm{Hz} / \mathrm{ppm}$ or $\mathrm{Hz} / \mathrm{vol} \%$, which represents the degree of change in response to the concentration of a certain analyte. Sensor selectivity refers to the ability of the sensor to distinguish a certain species in the presence of multiple analytes. The detection limit of gas sensors or the limit of detection (LOD) is the minimum amount of gas a sensor can detect. Response time and response recovery refer to the adsorption and desorption speeds of a sensor with respect to the detected analyte, respectively. These properties vary according to the type of sensing material used in the gas sensor and the mechanism of detection. 
There are many types of gas sensor developed to fulfill many roles. The calorimetric gas sensor, for example, is based on calorimetry as the transduction principle, in which the heat of a reaction is measured on the sensor surface. The calorimetric gas sensor has evolved from a simple platinum hot wire to the micro-electro-mechanical system (MEMS). ${ }^{(19)}$ The basic operation of the calorimetric gas sensor is to detect the change in heat from combustible gases on the sensor surface. ${ }^{(19,20)}$ Catalytic, combustible, and thermometric gas sensors are various configurations of the calorimetric gas sensor. The catalytic gas sensor may be one of the oldest sensors used to detect combustible gases, such as methane. It normally consists of a detector element (D), which contains a catalytic material that is sensitive to combustible gases and also an inert compensator element (C), as shown in Fig. 3(a). The catalytic gas sensor requires air or oxygen gas in order to function properly and it is also susceptible to contamination by the catalyst in its reaction with certain gases. ${ }^{(19)}$ On the other hand, thermal conductivity gas sensors can be used to detect gases with higher thermal conductivity than air such as hydrogen gas or methane gas [Fig. 3(b)]. A gas sample diffuses through a porous membrane into the sample chamber and is examined by comparing the rate of heat loss. However, gases such as ammonia and carbon monoxide, both with thermal conductivities similar to that of air, cannot be detected through this method.

In clean air

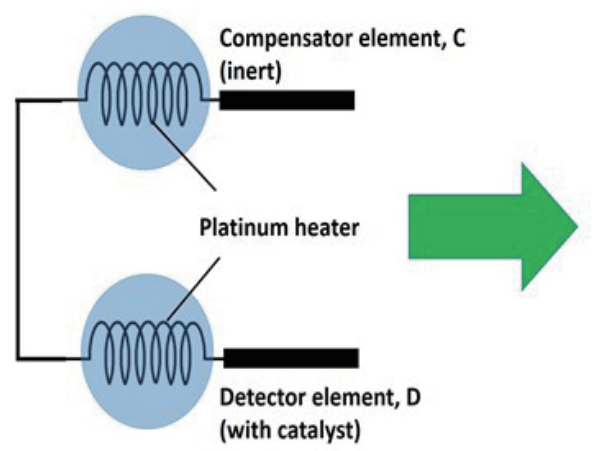

In combustible gases

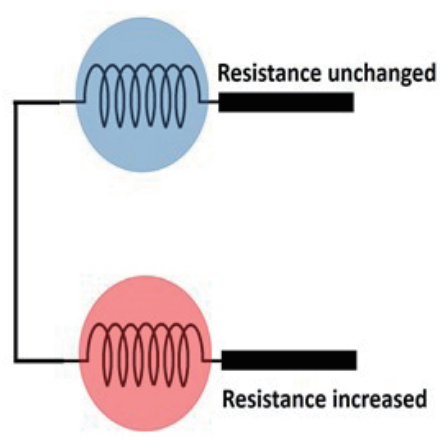

(a)

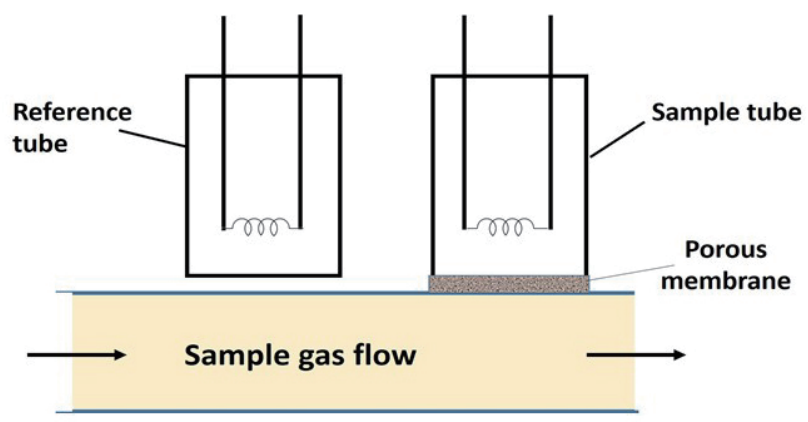

(b)

Fig. 3. (Color online) Basic principles of (a) catalytic and (b) thermal conductivity gas sensors. 
The optical gas sensor developed using a fiber optic technique is based on optical absorption or scattering of the gas analyte at a defined optical wavelength. The optical fiber is a small and lightweight device, but detection measurement can be easily distorted with interruption from ambient light interference. ${ }^{(21)}$ This sensor can be costly compared with many conventional gas sensors. In 1991, Philip Russell proposed a special optical fiber combining the properties of a photonic crystal with conventional optical fibers. The photonic crystal fiber (PCF) has been explored as a versatile sensor of gaseous, liquid, and even solid materials. ${ }^{(21,22)}$ Rifat et al. have proposed a surface plasmon resonance (SPR) sensor-based PCF design using a graphene-silver coating. ${ }^{(22)}$ It is said that the graphene coating can improve sensing performance by providing a high surface-to-volume ratio, better absorption of analytes, and superior plasmonic properties, while also inhibiting the oxidation of silver. ${ }^{(23)}$ The large enhancement of the plasmonic signal by gold-decorated graphene may be beneficial to the optical gas sensor. ${ }^{(23)}$ Graphene aeorogel, a three-dimensional (3D) ultralightweight material $\left(0.2 \mathrm{~g} \mathrm{~cm}^{-3}<\rho<3 \mathrm{~g} \mathrm{~cm}^{-3}\right)$, can absorb up to three times its own weight in oil. ${ }^{(24)}$ The aerogel is highly compressible and its combination with piezoelectric $\mathrm{SnO}_{2}$ or $\mathrm{GaN}$ nanoparticles provides a good pressure sensor. ${ }^{(24)}$ These properties of graphene aerogel can be explored for a wide class of piezoelectric or acousticwave-based gas sensors, such as surface acoustic wave (SAW), quartz crystal microbalance (QCM) or bulk acoustic wave (BAW), and cantilever-based devices.

The electrochemical gas sensor allows an analyte gas to pass through a membrane at an electrode where it is reduced or oxidized. ${ }^{(25)}$ It measures gases at relatively low concentrations and is capable of detecting a wide range of different gases. The electrochemical gas sensor can be in either a two- or three-electrode configuration. The three-electrode configuration consists of a working or sensing electrode, a counter electrode, and a reference electrode. The sensing principle of a simple two-electrode configuration is shown in Fig. 4. The sensing material deposited on the electrode reacts with analytes also functioning as ion and electron conductors. The semiconductor metal oxide sensing material translates this interaction into changes in

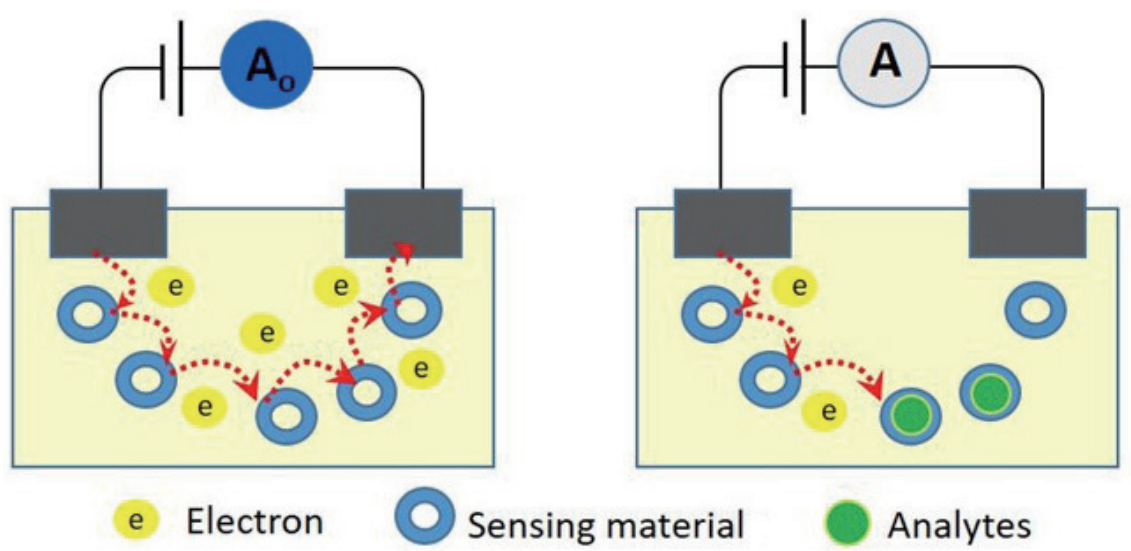

Fig. 4. (Color online) Basic principle of electrochemical gas sensor where the sensing material serves as a medium to trap the analyte and induces a current change in the external circuit. The left figure shows the circuit in the absence of target gas analytes and the right figure shows the circuit in the presence of gas analytes. 
electronic charge density and charge carrier mobility. ${ }^{(25)}$ As a result, there is a change of the electrical signal. Most electrochemical gas sensors are amperometric sensors, where the change in current indicates the rate of the electrolytic process in the sensing material. ${ }^{(25)}$

In recent years, there have been a significant number of publications regarding the use of graphene as an electrochemical gas sensor. The incorporation of graphene enhances the properties of conventional sensing materials by transferring its unique properties into the sensor. For example, the large specific surface area of graphene provides many possible paths for a molecule to be detected. ${ }^{(16,26)}$ Every atom in a single graphene layer is a possible adsorption site, making it an ultrasensitive material for detecting gas molecules. ${ }^{(26)}$ Graphene atoms and adsorbed gas molecules can interact via weak van der Waals interaction, hydrogen bonding, charge transfer, and strong covalent bonding. ${ }^{(26,27)}$ These interactions lead to changes in freeelectron concentration and a Dirac point shift, which can be measured by an electronic system. ${ }^{(27)}$ In chemically modified graphene (GO and reduced GO), the presence of surface defects can improve the absorption efficiency of gas molecules by allowing anchoring capability for many types of chemical functionality. ${ }^{(27,28)}$ Modification of graphene such as by sulphur doping can establish a bandgap in normal zero-gap semiconductor graphene. The presence of the bandgap leads to a change in carrier concentration in graphene when in contact with polluting gas molecules such as $\mathrm{NO}$ and $\mathrm{NO}_{2}{ }^{(29,30)}$ The compatibility of graphene with biomolecules, such as an enzyme via noncovalent tethering, gives selectivity to gas sensing via molecular recognition. ${ }^{(31)}$ The properties of nitrogen-doped graphene electrochemical biosensors are studied by redox peak current analysis in cyclic voltammetry measurement. ${ }^{(32)}$ Nevertheless, both sulphur- and nitrogen-doped graphene have been considered difficult to synthesize.

For now, an electrochemical strategy is the most convenient approach to study changes in the chemical environment of graphene. ${ }^{(33)}$ In graphene-based gas sensors, the interface charge layer is modified by the adsorption of different gas molecules. ${ }^{(34)}$ The corresponding electrical signal varies with the type of gas analyte used. Analytes with electron donors increase the current. Ion-polar analytes, on the other hand, disturb the charge transport and reduce the amount of current in graphene. Other than current, electrical characteristics, such as resistance and capacitance, can be measured upon the detection of analytes. In the future, graphene perhaps may have a large impact on electronic devices, and discussion on graphene-based sensors is therefore desirable for industrial, environmental, public safety, and military applications. ${ }^{(29,35)}$

\section{Synthesis of Graphene}

Various methods for producing different types of graphene material have been introduced owing to the need to modify graphene for specific applications, such as in optoelectronics, electronics, sensors, and many more. Methods such as chemical vapor deposition (CVD), mechanical exfoliation/cleavage of natural graphite, electric arc discharge, epitaxial growth, unzipping of CNTs, and solution-based reduction of GO are well known. ${ }^{(36-39)}$ Many have agreed that graphene is most suitable for electronic purposes as a $2 \mathrm{D}$ crystal consisting of not more than 10 graphene layers; ${ }^{(40)}$ more than these, the thin film has a much more complicated 3D electronic band structure. Thus, choosing the suitable graphene synthesis process should be approached with utmost consideration. The Nobel Prize winners Geim and Novoselov 
discovered graphene sheets after the mechanical exfoliation of highly oriented pyrolytic graphite (HOPG), a method now known as the Scotch tape method. ${ }^{(12)}$ The extraction of graphene sheets from well-dispersed graphene in a solvent is also an attractive research topic because of the stability issue of graphene during potential device fabrication. Problems such as the reduction of GO in water, which usually results in aggregated graphene, make it difficult for further redispersion in organic solvents since graphene is hydrophobic and GO is hydrophilic in nature. ${ }^{(41)}$ There is also a concern that the large-scale production of high-quality graphene that could be costly and harmful to the environment.

\subsection{Mechanical cleavage}

Micromechanical cleavage, popularly known as the Scotch tape method, is one of the bestknown methods of producing graphene sheets. ${ }^{(42)}$ Original researchers placed adhesive tape over graphite flakes and gradually reduced them to thinner layers. ${ }^{(12)}$ The steps of peeling with an adhesive tape placed over graphite are shown in Fig. 5. The peeled off tape containing layers of graphene is dissolved in a solution and later transferred onto a silicon wafer. ${ }^{(12)}$ A slightly different form of the mechanical method involves pushing bulk graphite along a silicon surface. As a result, graphene sheets are transferred to the rubbed silicon surface. In both cases, silicon is chosen as the preferred surface because it provides enough optical contrast with graphene for the human eye to detect without the aid of an optical microscope. ${ }^{(29)}$ Attempts to mechanically exfoliate graphite typically only result in stacks of graphene sheets or a few isolated sheets in low yield. ${ }^{(43)}$ Generally, graphene sheets up to $100 \mu \mathrm{m}$ in size or with 20 to 100 layers in thickness can be produced for most research purposes. ${ }^{(29)}$ Mechanical exfoliation needs to be performed with ultrasonication in liquids in order to overcome the van der Waals forces between sheets. ${ }^{(44)}$ The few-layer graphene (FLG) method based on mechanical exfoliation can provide high-scale graphene with a thickness not more than 10 graphene layers, but the electronic features of FLGs have been found to be 3D, thus limiting its electronic application. ${ }^{(44)}$ Graphene layers can be measured by Raman microscopy or electron microscopy techniques, such as high-resolution tunneling electron microscopy (HRTEM).

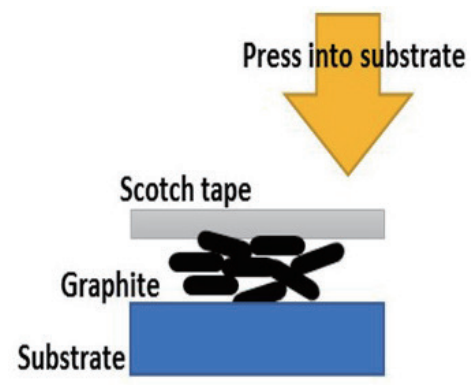

(a)

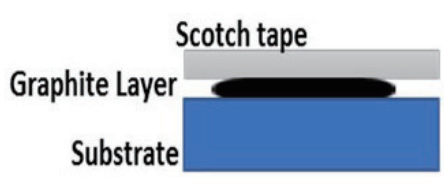

(b)

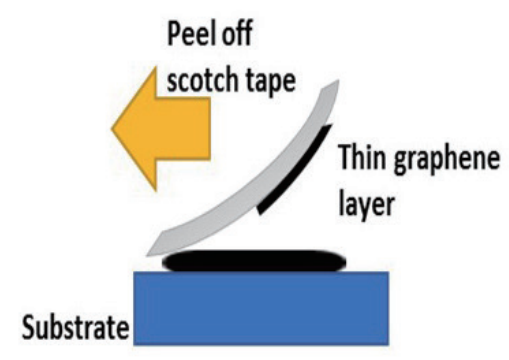

(c)

Fig. 5. (Color online) Schematic illustration of the Scotch tape exfoliation method for graphite to form graphene. Steps (a) to (c) are repeated until the graphite is reduced to a thin layer of graphene on the substrate. 


\subsection{CVD}

CVD is a thermal deposition method, by which single-layer graphene and FLG are grown from hydrocarbons on a substrate, such as silicone $(\mathrm{Si})$, nickel $(\mathrm{Ni})$, copper $(\mathrm{Cu})$, or ruthenium $(\mathrm{Ru}){ }^{(29)}$ The starting material of graphene is delivered as a hydrocarbon source (in vapor, usually methane) in a pressured gas flow and the targeted metal substrate is subjected to a very high temperature $\left(800-1100{ }^{\circ} \mathrm{C}\right.$ ) (Fig. 6). Along with methane, a small amount of hydrogen is added to the carrier gas to control the growth environment. After some time, the product of the catalysis of the metal and methane forms single-layer graphene on the substrate. ${ }^{(45)}$ Other than methane, graphene can also be synthesized from other carbon sources, such as polymer poly(methyl methacrylate) (PMMA), using CVD. ${ }^{(46)}$ Graphene processed by CVD can be fabricated into a large sheet film but with a limited number of layers (no more than several layers) that is applicable to many thin-film electronic device applications. ${ }^{(45,47,48)}$ Homogenous monolayer graphene possesses a defect-free, highly conductive network required for efficient charge transfer. ${ }^{(29)}$ CVD has been used for fabricating graphene-based gas sensors. A graphene film is initially grown on a copper substrate by CVD using a mixture of methane and hydrogen gases at a ratio of 5:95. ${ }^{(34)}$ The graphene film is then transferred to a $\mathrm{SiO}_{2} / \mathrm{Si}$ substrate and a 50 -nm-thick gold layer is coated onto the transferred graphene film by vacuum evaporation. The weakness of a CVD-grown graphene device is its susceptibility to contamination during the transfer of the graphene film. ${ }^{(31)}$ Another form of thermal fabrication of graphene is by epitaxial growth on silicon carbide ( $\mathrm{SiC})$, which has been a popular route to produce highquality monolayer graphene. ${ }^{(29,45)}$ Edward Goodrich Acheson discovered the formation of pure crystalline graphite after $\mathrm{SiC}$ was heated to a temperature of more than $4000{ }^{\circ} \mathrm{C}$. Epitaxial growth is a well-known method for producing SiC-graphene gated electronics. Both CVD and epitaxial growth of graphene require specialized equipment and have disadvantages in terms of cost, making them uneconomical for practical and industrial purposes. ${ }^{(45)}$

\subsection{Chemical oxidation-reduction method for graphite}

A chemical method, specifically chemical reduction of GO, is favorable for large-scale production because of its efficient approach; however, the electronic properties of graphene produced via chemical means are inferior to those obtained by CVD and mechanical

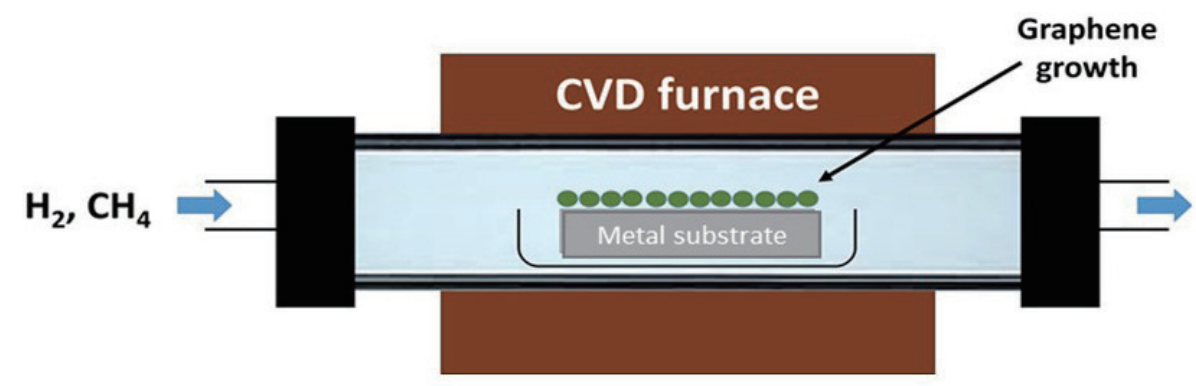

Fig. 6. (Color online) Schematic illustration of CVD growth process for easy control of graphene layer formation. 
exfoliation. ${ }^{(49-53)}$ Unlike in the previous methods, graphene synthesized by a chemical oxidation-reduction method exhibits many structural defects. ${ }^{(54)}$ The oxidation of graphite to GO has been shown to increase the distance between adjacent sheets from $3.35 \AA$ in graphite to $6.8 \AA$ in $\mathrm{GO} .{ }^{(55)}$ The intercalation of chemical species within graphite oxide layers facilitates exfoliation, which needs to overcome the van der Waals force between the graphitic layers in order to produce separate GO sheets. Highly reduced graphene oxide (rGO) with graphene domains, defects, and residual oxygen-containing groups on each sheet is obtained after reduction of $\mathrm{GO}$, which involves the elimination of oxygen-containing groups using reducing agents such as hydrazine. ${ }^{(56,57)}$ The synthesis route of $\mathrm{rGO}$ by the oxidation of graphite by Hummer's method to produce GO, further exfoliation to form separate GO sheets, and finally the reduction of GO is depicted in Fig. 7.

Hummer's method utilizes potassium permanganate $\left(\mathrm{KMnO}_{4}\right)$ as the oxidant in a mixture of graphite powder with sodium nitrate $\left(\mathrm{NaNO}_{3}\right)$ and concentrated sulfuric acid $\left(\mathrm{H}_{2} \mathrm{SO}_{4}\right)$, which is a safer approach than the use of fuming nitric acid $\left(\mathrm{HNO}_{3}\right)$ and potassium perchlorate $\left(\mathrm{K}_{2} \mathrm{ClO}_{3}\right){ }^{(58-61)}$ Hummer's method, however, produces in situ $\mathrm{HNO}_{3}$ and induces contamination with excess permanganate ions. Many alternatives have been proposed to improve Hummer's method because of these environmental concerns. ${ }^{(62,63)}$ The distribution of oxygen-containing functional groups of GO [for example, hydroxyl and epoxy (1,2-ether) groups, carbonyl groups, carboxylic acids, and organic carbonyl defects] has been an interesting topic for many years, ${ }^{(64)}$ but these functional groups are clearly important as reactive handles for various surface modification reactions.

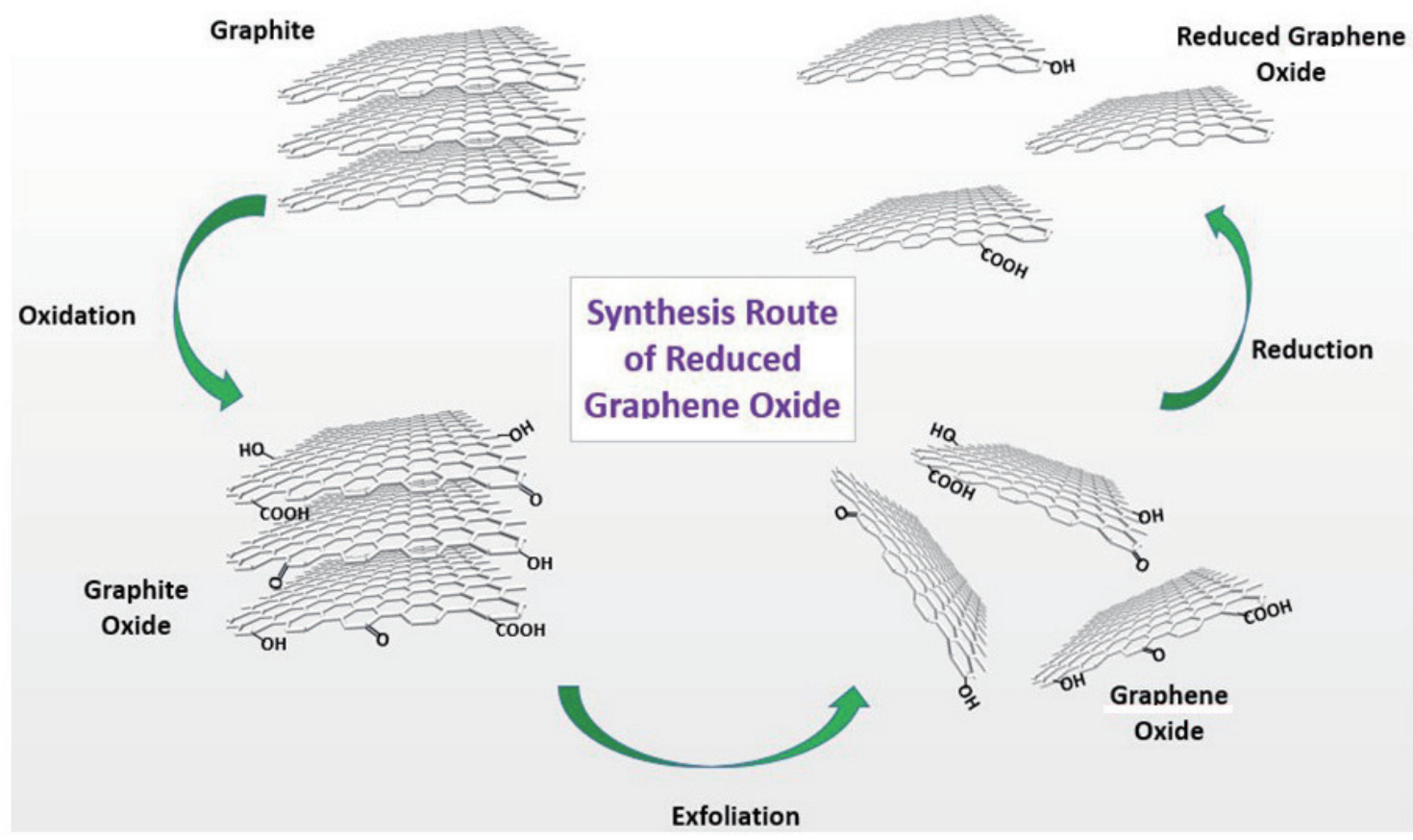

Fig. 7. (Color online) Typical synthesis route of rGO from graphite using Hummer's method. 
On the other hand, rGO has shown potential for a range of applications including conductive layers, nanoelectronics, sensors, and energy storage. ${ }^{(28)}$ The advantage of rGO over monolayer defect-free graphene is the presence of dangling oxygen functional groups on the graphene surface and edges. ${ }^{(65)}$ Both theoretical and experimental results revealed that tuning of the oxygen functional groups on rGO plays a vital role in the detection of several organic compounds. Experimentally, oxygen functional groups can be tuned by adjusting the reduction time, the type of reducing agent, and the environment of reduction. rGO with a high number of oxygen functional groups produced in a short reduction time shows a faster response to analytes. A rapid electrical response to many gases or vapors has been reported for rGO reduced using ascorbic acid. ${ }^{(28)}$

\section{Application of Graphene in Electrochemical Gas Sensor}

Electrochemical sensors exhibit advantages over other types of sensor because of their widerange tunability (in terms of electrode functionality), sensitivity toward analytes present within the system, and economical and easy application. Normally, the graphene film is placed as an electrode, and once it interacts with oxidizing analytes, it results in the depletion of charge carriers in the system. The opposite occurs when graphene interacts with reducing analytes. ${ }^{(66,67)}$ Physisorbed analytes can also be measured using an electrochemical system. Functional groups on graphene may have larger effects on this mechanism as electrical responses to chemical vapors are seen to diminish by 1-2 orders of magnitude after the elimination of functional groups. ${ }^{(67)}$ A graphene electrode is already superior to indium tin oxide (ITO) in spectroelectrochemistry applications. ${ }^{(64)}$ However, it should be considered that electrochemical properties of graphene also vary with its molecular structure and composition, and the way it is synthesized as graphene nanoplatelets (GNPs), nanoribbons, or fibers.

\subsection{Detection of common organic vapors}

Common organic vapors are produced in large quantities in industry. Their production should be monitored because they are toxic, vaporize swiftly, and can easily escape to surrounding areas. The first sensors for the detection of organic vapor were developed in the early 1920s by James Sumner to detect methanol and formaldehyde. ${ }^{(68)}$ Acetone is a selective breath marker, and the prolonged presence of a certain concentration of acetone may reflect diabetes. $^{(69)}$ Ammonia and toluene are now common indoor air pollutants. Ammonia is produced by hydrolysis of urea in antifreeze additives in concrete buildings. ${ }^{(67,70)}$ Toluene, which can be found in the production lines of paints, metal cleaners, plastics, and detergents, is an indispensable cancer biomarker. ${ }^{(71,72)}$

A field-effect transistor (FET) is a type of device architecture often used to explore the full potential of nanomaterials. The electrical response of graphene FET-based gas sensors cannot provide the desired performance when it comes to the detection of ammonia, as demonstrated by Dan et al., ${ }^{(67)}$ because of the weak binding $(\sim 20 \mathrm{meV})$ and small charge transfer for 
ammonia on graphene. On the other hand, metal oxide nanoflower-decorated rGO has excellent sensing performance and stability for formaldehyde and ammonia gas sensing. ${ }^{(70)}$ The origin of the sensing mechanism is the depletion layer formed at the metal oxide-rGO heterojunction that can be modulated by the diffused gas molecules, which also serve as a donor of negative charges (for ammonia). The metal oxide prevents further aggregation of rGO sheets, hence giving a higher surface area and active sites to the sensing electrode, and a higher stability of the sensor array.

In chemically reduced graphene, the role of the reducing agent has been investigated in all-organic vapor sensors. GD is typically reduced using ascorbic acid as a mild and green reducing agent. ${ }^{(28)}$ An aniline monomer was used to reduce GO in order to study the gas sensing performance of different states of polyaniline attached to $\mathrm{rGO} .{ }^{(73)}$ The chemically modified rGO not only exhibited a higher sensitivity but a specific higher sensing response to ammonia than to other vapors. A similar finding was reported by another group that used pyrrolereduced GO for ammonia detection at room temperature. ${ }^{(74)}$ Their pyrrole-modified rGO was observed to exhibit a large resistance change in a short response time of $1.4 \mathrm{~s}$ measured at an ammonia concentration of $1 \mathrm{ppb}$. This is due to the intrinsic properties of rGO sheets as well as the adsorbed polypyrrole molecules. Aniline and pyrrole are monomers of highly conducting polymers with polypyrrole having a higher electron affinity. From both studies, it can be concluded that the method of preparing chemically reduced $\mathrm{rGO}$ is crucial in determining the sensing performance, because graphene film morphology varies according to the concentration of the conducting polymer. If the polymer completely covers the graphene, the interactions between ammonia gas and $\mathrm{sp}^{2}$-bonded carbon are hindered, thus giving a smaller sensing response.

As discussed previously, rGO consists of oxygen functional groups partially decorated on the graphene sheets that act as active sites to the gas molecules. As reported by Minitha et al., the concentration of oxygen functional groups of rGO can be controlled by the aging time during the reduction process with hydrazine hydrate. ${ }^{(65)}$ A higher concentration of oxygen functional groups yields a higher sensing response but poor recovery of the sensor. The sensor has difficulty in recovering its initial state since there are now more anchored functional groups in the graphene sheet, thus the recovery treatment should be several times higher. Theoretical calculation shows that rGO responds more sensitively to toluene than ammonia owing to toluene having a higher binding energy. Charge transfer from toluene to rGO is easier owing to the existence of an electron-donating methyl group attached to the benzene ring of toluene.

rGO ink exhibits remarkable performance as a state-of-the-art wireless gas sensor. By inkjet printing on flexible substrates (paper and Kapton), the gas sensor developed by Le et al. showed an approximately $10 \Omega$ change in resistance within 1 min after introducing ammonia. ${ }^{(75)}$ In comparison with other works on graphene-based gas sensors, their inkjet-printed rGO demonstrated a short recovery time without requiring UV or heat treatment as reported in Refs. 73 and 74. An inkjet-printed-rGO gas sensor based on the same technology fabricated on poly(ethylene terephthalate) is capable of the reversible detection of nitrogen dioxide $\left(\mathrm{NO}_{2}\right)$ and chlorine $\left(\mathrm{Cl}_{2}\right)$ gases at a ppb sensitivity level. ${ }^{(28)}$ 


\subsection{Detection of toxic gases}

Various toxic gases lead to different changes in the electrical properties of graphene upon adsorption on its surface. Even though the charge transfer mechanism may be a dominant factor in these changes, the same cannot be said for all analytes. The electrical conductivity of rGO increases upon $\mathrm{NO}_{2}$ gas adsorption, whereas the adsorption of hydrogen gas $\left(\mathrm{H}_{2}\right)$ and methane gas $\left(\mathrm{CH}_{4}\right)$ decreases the electrical conductivity of rGO ${ }^{(17)}$ The former is caused by the electron withdrawing effect of the adsorbed $\mathrm{NO}_{2}$, which leads to more positive charge carriers (or holes) in p-type rGO. However, in the latter, $\mathrm{H}_{2}$ or $\mathrm{CH}_{4}$ is neither an electron donor nor an electron acceptor; thus, it is not capable of charge transfer. Instead, the gas molecules from synthetic air that previously physisorbed on the surface of rGO are replaced by $\mathrm{H}_{2}$ or $\mathrm{CH}_{4}$ molecules, which are responsible for the observed electrical changes. The same goes for the deceases in resistance when $\mathrm{NO}_{2}$ and ammonia $\left(\mathrm{NH}_{3}\right)$ are adsorbed on graphene as they both induce hole and electron conduction, respectively. This similarity is due to graphene initially having no carriers in its conduction band, and measurements such as Hall effect measurement can reveal the nature of charge carriers in graphene. ${ }^{(18)}$

The poor selectivity of a graphene sensor can be improved by chemical functionalization and doping of metal nanoparticles and metal oxides. ${ }^{(17)}$ It has been shown that the resistance of a palladium (Pd)-doped GO-based sensor changes because of the shift in the work function of Pd upon $\mathrm{H}_{2}$ gas adsorption. ${ }^{(76)}$ The dissociative chemisorption process is selective to $\mathrm{H}_{2}$ gas only, with no measurable signal response toward other analytes, such as toluene, carbon monoxide, and ethanol. Similarly to $\mathrm{NO}_{2}$, the electrons from graphene are transferred to sulfur dioxide $\left(\mathrm{SO}_{2}\right)$, which acts as an electron acceptor so that the increase in conductance upon adsorption can be measured. ${ }^{(77,78)}$

Designing a hybrid heterostructure nanofunctional material as a sensing electrode may be the most efficient approach for a high-sensitivity gas sensor. A recently published work by $\mathrm{Hu}$ et al. explained how an rGO-carbon dot (CD) hybrid achieved a 3.3-fold higher sensitivity toward $\mathrm{NO}_{2}$ than a bare rGO sensor device. ${ }^{(79)}$ The $\mathrm{CD}$, also being a $2 \mathrm{D}$ electronic material, gives a higher hole carrier density at the surface of rGO and eventually promotes charge transfer. The green one-pot synthesis method includes the reduction of GO and the in situ generation of CDs on the rGO' surface. Unlike the other types of heterojunction that form with unequal crystal structures and bandgaps, a CD-rGO hybrid device contains a 3-8-nm-size CD with a similar lattice parameter of graphene, thus resulting in more efficient charge transfer. Hybrid rGOcopper pthalocyanine (CuPC) nanoflowers was explored as sensing material for detection of chlorine gas. The sensor detection limit was measured to be $1.97 \mathrm{ppb}$ at room temperature. ${ }^{(80)}$ The physisorption of $\mathrm{Cl}_{2}$ molecules occurred on the central metal ion present on the grains of CuPc-decorated rGO. Table 1 shows the sensing responses of several graphene-based gas sensors for some toxic gases.

\subsection{Detection of chemical warfare agent simulant}

Comparable to biological and nuclear warfare agents, chemical warfare agents (CWAs) are considered to be one of the most lethal types of weapon to be created by humanity as they 
Table 1

Sensing responses of graphene-based sensors toward certain toxic gases.

\begin{tabular}{lccccc}
\hline Material & Method of detection & Target gas & LOD & Temperature $\left({ }^{\circ} \mathrm{C}\right)$ & Reference number \\
\hline rGO + octadecylamine & Chemiresistor & $\mathrm{NO}_{2}$ & $0.3 \mathrm{ppm}$ & 80 & 17 \\
rGO + CD & Amperometry & $\mathrm{NO}_{2}$ & $10 \mathrm{ppb}$ & $\mathrm{RT}$ & 79 \\
$\mathrm{rGO}$ & Chemiresistor & $\mathrm{SO}_{2}$ & $5 \mathrm{ppm}$ & 27 & 77 \\
$\mathrm{GO}$ & FET & $\mathrm{SO}_{2}$ & $275 \mathrm{ppm}$ & $\mathrm{RT}$ & 78 \\
$\mathrm{rGO}-\mathrm{CuPC}$ & Impedance & $\mathrm{Cl}_{2}$ & $1.97 \mathrm{ppb}$ & $\mathrm{RT}$ & 80 \\
rGO-CuPd & Chemiresistor & $\mathrm{H}_{2}$ & $50 \mathrm{ppm}$ & 35 & 76 \\
\hline
\end{tabular}

are relatively inexpensive and easy to produce. CWAs can be dispersed as a gas, liquid, and aerosol, and as agents adsorbed on powder particles. Some examples of CWAs are sarin, soman, sulfur mustard, nitrogen mustard, and VX. ${ }^{(81)}$ Their use in conventional research laboratories is restricted; thus, the research on CWAs is usually conducted using simulants. Apart from the fundamental toxicological properties, simulants mimic all relevant properties of CWAs. ${ }^{(82,83)}$ Mustard gas is a cytotoxic synthetic chemical belonging to the blister agent class of CWAs. ${ }^{(84)}$ Some of the simulants, such as thiodiglycol (TDG) and chloroethyl methyl sulfide, are shown in Fig. 8. Dimethyl methylphosphonate (DMMP), an organophosphate compound, is another popular CWA simulant that represents G-series agents [tabun (GA), sarin (GB), soman (GD)] (Fig. 9). It is a type of nerve agent that disrupts the balance between sympathetic and parasympathetic autonomic nervous systems upon exposure. ${ }^{(85)}$ Figure 10 shows the structures of V-series agents and their simulants. V-series agents persist in nature, whereas G-series agents are volatile.

In the environment, the hydrolysis of sulfur mustard leads to the formation of TDG, a polar and nonvolatile compound. There is no electrochemical activity of a simulant molecule on a bare electrode; hence, the electrode must be modified with catalysts. Singh et al. first reported the employment of GO as a carbo-electrocatalyst, commonly called carbon electro-catalyst, for the electrochemical oxidation of TDG at room temperature. ${ }^{(86)}$ Previously, transition metals were used as catalysts for electrochemical oxidation. Electrocatalytic performance was evaluated using cyclic voltammetry and differential pulse voltammetry measurements. 2-(2-Hydroxy-ethanesulfinyl)-aldehyde was identified as the main oxidation product of TDG. An electrochemical sensor for sulfur mustard gas was developed by employing the choline oxidase enzyme as a sustainable sensing tool. ${ }^{(87)}$ This bioassay sensor measured the enzymatic by-product hydrogen peroxide in the chronoamperometric mode on a modified graphite-based electrode or screen-printed electrode (SPE). Biological molecules, such as enzymes, antibodies, and DNAs, are the pathway for selective detection via single-target-substrate capture. Enzymes deliver fast detection and have a high rate of reusability. A commercially available miniaturized SPE is an example of how a graphene electrode can be employed during on-site gas detection where a biological material sensing tool can be anchored.

A study of GO reduced with p-phenylenediamine (PPD) showed the detection of lowconcentration DMMP $(10 \mathrm{ppm}) .{ }^{(28)}$ The resistance of the device was seen to increase upon adsorption without saturation. The stacked structure of rGO nanosheets allowed the DMMP gas molecules to move rapidly across the membrane so that the sensor could recover to its original 


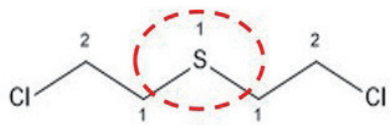

Mustard gas (1-Chloro-2-[(2-chloroethyl)sulfanyl]ethane)

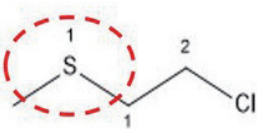

Chloroethyl methyl sulfide

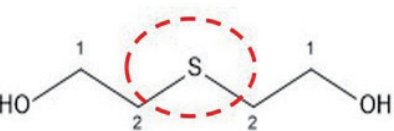

2-(2-Hydroxyethylsulfanyl)ethanol or thiodiglycol (TDG)

Fig. 8. (Color online) Chemical structures of mustard gas and its simulants. Dashed circles show the similar chemical structures between the CWA and its simulants.

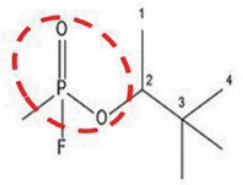
Soman (3,3-Dimethylbutan-2-yl
methylphosphonofluoridate)

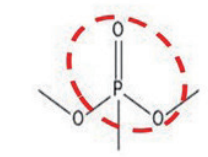

Dimethyl

methylphosphonate trimethylphosphate

(DMMP)

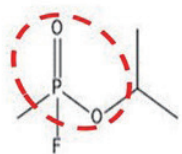

Sarin (Propan-2-yl methylphosphonofluoridate)

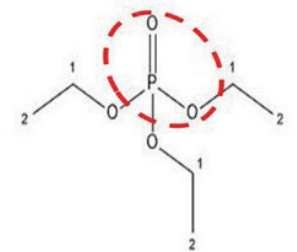

triethylphosphate

Fig. 9. (Color online) Chemical structures of soman, sarin, and tabun, and their possible simulants. Dashed circles show the similar chemical structure between the CWA and its simulants.

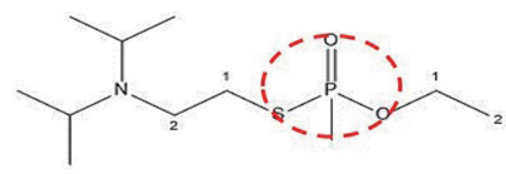

VX (Ethyl (\{2-[bis(propan-2-yl)amino]ethyl\}sulfanyl)(methyl)phosphinate)

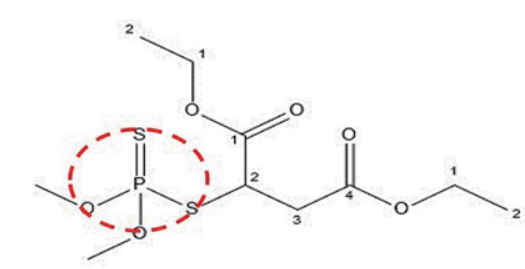

Malathion (Diethyl 2 [(dimethoxyphosphorothioyl)sulfanyl]butanedioate)

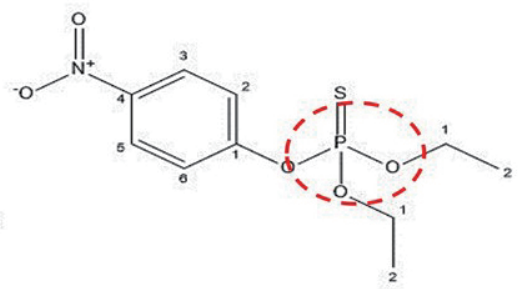

Parathion (O,O-Diethyl O-(4-nitrophenyl) phosphorothioate)

Fig. 10. (Color online) Chemical structures of VX agents and their possible simulants. Dashed circles show the similar chemical structure between the CWA and its simulants. 
state for repeatability. The charge transfer occurred between DMMP and graphene because DMMP is a strong electron donor, similar to $\mathrm{NO}_{2}$. Also, for DMMP gas detection, Hwang et al. fabricated a 3D mesoporous structure of nonstacked rGO-hexafluorohydroxypropanyl benzene (HFHPB) nanosheets as a sensing material. ${ }^{(88)}$ A porous material is desirable when rapid and sensitive gas detection is needed. The nonstacked rGO has 53 -fold greater porosity than the other carbon materials. The electron withdrawing trifluoromethyl group of HFHPB is the receptor for the organophosphate group of DMMP via hydrogen bonding. The large pore size $(40.37 \mathrm{~nm})$ and high pore volume $\left(5.1219 \mathrm{~cm}^{3} \mathrm{~g}^{-1}\right)$ of the hybrid film contributed to easier and faster diffusion of DMMP into the large exposed rGO surface area $\left(507.5 \mathrm{~m}^{2} \mathrm{~g}^{-1}\right)$ for detection to occur. The HFHPB group was chemically grafted to the nonstacke $\mathrm{rGO}$ via the diazotization reaction.

GNPs can be produced in large quantities and are economical for the production of singlelayer graphene. ${ }^{(89)}$ This motivated Wiederoder et al. to investigate the selective detection of CWA simulants using a polymer-GNP composite electrode. ${ }^{(90)}$ A set of diverse polymers were prepared for the detection of several different G-series simulants, such as Nafion for DMMP, polyvinyl alcohol (PVA) for trimethyl phosphate (TMP), and polyisobutylene (PIB) for trielthyl phosphate (TIP). A highly accurate result was obtained, but the sensing mechanism was not explained.

Another form of detection is to quantitatively measure the capability of hydrogen bonding between GO and the CWA simulant. For example, DMMP contains phosphoryl $(\mathrm{P}=\mathrm{O})$ groups, that give stronger hydrogen bonds to any available surface. ${ }^{(91)}$ On the GO surface, oxygen functional and modified functional groups are available to form hydrogen bonds with DMMP. A study of the chemical interaction of DMMP and a self-assembled interface by Bertilsson et al. showed that an acid surface $(-\mathrm{COOH})$ has the strongest DMMP adsorption. ${ }^{(92)}$ However, it is difficult to use electrochemistry to probe weaker noncovalent interactions (hydrogen bonding, electrostatic, hydrophobic, $\pi-\pi$, van der Waas) during molecular physisorption owing to the fact that the work function of conventional metal electrodes cannot be changed. For that purpose, Kulkarni et al. reported the molecular dipole detection of an extremely low DMMP concentration of $0.64 \mathrm{ppb}$ using a graphene nanoelectronic heterodyne sensor (alternating current signal detection). ${ }^{(93)}$ Their instruments probed the interaction between graphene and polar analytes using a graphene FET as a high-frequency mixer with a surface-adsorbed analyte functioning as an oscillating gate. From their observation, polar molecules yield a stronger signal and nonpolar molecules have a negligible signal.

Malathion, used as a simulant of V-series CWAs, is also a pesticide that can be found in many agricultural settings. In a study to improve the LOD of malathion, rGO, which served as a large adsorption surface for adsorption, was modified on the surface of polyamide and polypyrrole electrospun nanofibers. ${ }^{(94)}$ There has also recently been increased use of graphenebased electrodes made using a graphene ink and pencil graphite electrode (PGE). In one example, phosphotriesterase (PTE) was conjugated to the graphene surface via glutaraldehyde cross-linking and, as a result, rapid sensing with a response time of $5 \mathrm{~s}$ and an LOD of $3 \mathrm{nM}$ was obtained. ${ }^{(95,96)}$ It was hypothesized that the high sensitivity was achieved by the preparation of this heterogenous electrode with the assistance of laser annealing, which optimized the 
morphology of graphene as a template for biofunctionalization. Very detailed information on the preparation of this graphene electrode for this biosensor is described in Ref. 95.

\section{Conclusions}

Many prototypes and construction methods of graphene-based gas sensors have been proposed and reported over the last two decades given the importance of environmental monitoring, health, public safety, and national security. Owing to their unique electrical, physical, and chemical properties, graphene materials continue to drive the interest of researchers all over the world from various fields. Graphene-based sensors are cost-effective and easy to prepare through the use of chemical means such as Hummers' method and mechanical exfoliation. Two-dimensional graphene has superior electronic properties, but functional groups on the surfaces of $\mathrm{GO}$ and $\mathrm{rGO}$ have a major role in trapping and interacting with gas analytes. Graphene is available in many architectures and forms with different surface areas. The sensitivity of graphene-based gas sensors increases with increasing surface area for adsorption sites. A better understanding of the interaction mechanism between grapheneadsorbed analytes has been demonstrated in many previous electrochemical studies. In this review, we have discussed several attempts to achieve sensitivity and selectivity of sensors using graphene by incorporating conducting polymers, metals, and carbon dots that can form heterostructures with graphene. There is also growing interest in using molecular recognition as a selective sensing mechanism, such as that offered by biological species that can be noncovalently or covalently attached to graphene. Unfortunately, like many applications of graphene, the technological readiness of graphene-based gas sensors still lags behind flexible transparent conductors and photodetectors. The limitations of graphene are its stability in a humid environment and its sensor recovery speed. In conclusion, knowing the right graphene material and the way to optimize its use will lead to the commercializion of graphene as nextgeneration gas sensors.

\section{Acknowledgments}

The authors gratefully acknowledge the financial support from Chemical Defence Research Centre (CHEMDEF) for a research grant (UPNM/2018/CHEMDEF/ST/3). The authors would also like to thank the Ministry of Education of Malaysia and the government of Malaysia for research funding (ISIS-NEWTON/2019/SG/01).

\section{References}

1 A. Nag, S. C. Mukhopadhyay, and J. Kosel: Sens. Actuators A 251 (2016) 148. https://doi.org/10.1016/ J.SNA.2016.10.023

2 M. S. A. Rahman, S. C. Mukhopadhyay, P. Yu, J. Goicoechea, I. R. Matias, C. P. Gooneratne, and J. Kosel: J. Food Eng. 114 (2013) 346. https://doi.org/10.1016/J.JFOODENG.2012.08.026

3 A. I. Zia, S. C. Mukhopadhyay, I. H. Al-Bahadly, P. Yu, C. P. Gooneratne, and J. Kosel: Proc. 2014 IEEE Int. Instrumentation and Measurement Technology Conf. (IEEE, 2014) 838. https://doi.org/10.1109/ I2MTC.2014.6860861 
4 W. Tian, X. Liu, and W. Yu: Appl. Sci. 8 (2018) 1118. https://doi.org/10.3390/app8071118

5 A. Vaseashta, M. Vaclavikova, S. Vaseashta, G. Gallios, P. Roy, and O. Pummakarnchana: Sci. Technol. Adv. Mater. 8 (2007) 47. https://doi.org/10.1016/j.stam.2006.11.003

6 X. Liu, S. Cheng, H. Liu, S. Hu, D. Zhang, and H. Ning: Sensors 12 (2012) 9635. https://doi.org/10.3390/ s120709635

7 P. Sun, W. Zhao, W. Cao, Y. Guan, Y. Sun, and G. Lu: CrystEngComm 13 (2011) 3718. https://doi.org/10.1039/ c1ce05073g

8 M. Procek, A. Stolarczyk, T. Pustelny, and E. Maciak: Sensors 15 (2015) 9563. https://doi.org/10.3390/ s150409563

9 K. K. Sadasivuni, A. Kafy, H. Kim, H. Ko, S. Mun, and J. Kim: Synth. Met. 206 (2015) 154. https://doi. org/10.1016/J.SYNTHMET.2015.05.018

10 G. Neri: Chemosensors 3 (2015) 1. https://doi.org/10.3390/chemosensors3010001

11 A. Nag, A. Mitra, and S. C. Mukhopadhyay: Sens. Actuators A 270 (2018) 177. https://doi.org/10.1016/ j.sna.2017.12.028

12 K. S. Novoselov, A. K. Geim, S. V. Morozov, D. Jiang, Y. Zhang, S. V. Dubonos, I. V. Grigorieva, and A. A. Firsov: Science 306 (2004) 666. https://doi.org/10.1126/science.1102896

13 Physics Today: https://physicstoday.scitation.org/do/10.1063/PT.4.0866/full/ (accessed December 2019)

14 M. D. Stoller, S. Park, Y. Zhu, J. An, and R. S. Ruoff: Nano Lett. 8 (2008) 3498. https://doi.org/10.1021/ n1802558y

15 A. K. Geim and K. S. Novoselov: Nat. Mater. 6 (2007) 183. https://doi.org/10.1038/nmat1849

16 A. K. Geim: Science 324 (2009) 1530. doi: https://doi.org/10.1126/science.1158877

17 A. Zopfl, M. Lemberger, G. Ruhl, F. Matysik, and T. Hirsch: Faraday Discuss. 173 (2014) 403. https://doi. org/10.1039/C4FD00086B

18 J. D. Fowler, M. J. Allen, V. C. Tung, Y. Yang, R. B. Kaner, and B. H. Weiller: ACS Nano 3 (2009) 301. https:// doi.org/10.1021/nn800593m

19 R. E. Cavicchi: Calorimetric Sensors (Momentum Press, New Jersey, 2011).

20 N. Park, T. Akamatsu, T. Itoh, N. Izu, and W. Shin: Sensors (Basel) 14 (2014) 8350. https://doi.org/10.3390/ s140508350

21 J. F. Algorri, D. C. Zografopoulos, A. Tapetado, D. Poudereux, and J. M. Sánchez-Pena: Sensors 18 (2018) 4263. https://doi.org/10.3390/s18124263

22 A. A. Rifat, G. A. Mahdiraji, D. M. Chow, Y. G. Shee, R. Ahmed, and F. R. M. Adikan: Sensors 15 (2015) 11499. https://doi.org/10.3390/s150511499

23 G. R. S. Iyer, J. Wang, G. Wells, S. Guruvenket, S. Payne, M. Bradley, and F. Borondics: ACS Nano 8 (2014) 6353. https://doi.org/10.1021/nn501864h

24 M. Dragoman, L. Ghimpu, C. Obreja, A. Dinescu, I. Plesco, D. Dragoman, T. Braniste, and I. Tiginyanu: Nanotechnology 27 (2016) 475203. https://doi.org/10.1088/0957-4484/27/47/475203

25 I. Cretescu, D. Lutic, and L. R. Manea: Electrochemical Sensors for Monitoring of Indoor and Outdoor Air Pollution (Intech Open, 2017) http://dx.doi.org/10.5772/intechopen.68512

26 U. Latif and F. L. Dickert: Sensors 15 (2015) 30504. https://doi.org/10.3390/s151229814

27 Y. Zhu, S. Murali, W. Cai, X. Li, J. W. Suk, J. R. Potts, and R. S. Ruoff: Adv. Mater. 22 (2010) 3906. https:// doi.org/10.1002/adma.201001068

28 N. Hu, Y. Wang, J. Chai, R. Gao, Z. Yang, E. S. W. Kong, and Y. Zhang: Sens. Actuators B 163 (2012) 107. https://doi.org/10.1016/J.SNB.2012.01.016

29 A. K. Geim and A. H. Macdonald: Phys. Today 60 (2007) 35. https://doi.org/10.1063/1.2774096

30 H. Gao, Z. Liu, L. Song, W. Guo, L. Ci, A. Rao, W. Quan, R. Vajtai, and P. M. Ajayan: Nanotechnology 23 (2012) 275605. https://doi.org/10.1088/0957-4484/23/27/275605

31 Q. Zeng, J. Cheng, L. Tang, X. Liu, Y. Liu, J. Li, and J. Jiang: Adv. Funct. Mater. 20 (2010) 3366. https://doi. org/10.1002/adfm.201000540

32 H. Wang, T. Maiyalagan, and X. Wang: ACS Catal. 2 (2012) 781. https://doi.org/10.1021/cs200652y

33 Y. Guo, S. Guo, J. Ren, Y. Zhai, S. Dong, and E. Wang: ACS Nano 4 (2010) 4001. https://doi.org/10.1021/ nn100939n

34 M. Gautam and A. H. Jayatissa: Mater. Sci. Eng. C 31 (2011) 1405. https://doi.org/10.1016/J.MSEC.2011.05.008

35 S. Ammu: Sci. Lett. 4 (2015) 162.

36 B. G. Choi, H. Park, T. J. Park, M. H. Yang, J. S. Kim, S. Y. Jang, N. S. Heo, S. Y. Lee, J. Kong, and W. H. Hong: ACS Nano 4 (2010) 2910. https://doi.org/10.1021/nn100145x

37 T. Kuila, S. Bose, P. Khanra, A. K. Mishra, N. H. Kim, and J. H. Lee: Carbon 50 (2012) 914. doi: https://doi. org/10.1016/j.carbon.2011.09.053 
38 A. I. Kamisan, L. W. Zainuddin, A. S. Kamisan, T. I. T. Kudin, O. H. Hassan, N. A. Halim, and M. Z. A. Yahya: Key Eng. Mater. 708 (2016) 25. https://doi.org/10.4028/www.scientific.net/KEM.708.25

39 A. Z. Yazdi, K Chizari, A. S. Jalilov, J. Tour, and U. Sundararaj: ACS Nano 9 (2015) 5833. https://doi. org/10.1021/acsnano.5b02197

40 A. Gupta, T. Sakthivel, and S. Seal: Prog. Mater. Sci. 73 (2015) 44.

41 W. Chen, L. Yan, and P. R. Bangal: Carbon 48 (2010) 1146. https://doi.org/10.1016/j.carbon.2009.11.037

42 T. Kuila, S. Bose, P. Khanra, A. K. Mishra, N. H. Kim, and J. H. Lee: Biosens. Bioelectron. 26 (2011) 4637. https://doi.org/10.1016/j.bios.2011.05.039

43 O. C. Compton and S. T. Nguyen: Small 6 (2010) 711. https://doi.org/10.1002/smll.200901934

44 A. A. Pirzado, F. L. Normand, T. Romero, S. Paszkiewicz, V. Papaefthimiou, D. Ihiawakrim, and I. Janowska: ChemEngineering 3 (2019) 37. https://doi.org/10.3390/chemengineering3020037

45 K. E. Whitener and P. E. Sheehan: Diamond Relat. Mater. 46 (2014) 25. https://doi.org/10.1016/ j.diamond.2014.04.006

46 Z. Sun, Z. Yan, J. Yao, E. Beitler, Y. Zhu, and J. M. Tour: Nature 468 (2010) 549. https://doi.org/10.1038/ nature 09579

47 X. Liu, T. H. Metcalf, J. T. Robinson, B. H. Houston, and F. Scarpa: Nano Lett. 12 (2012) 1013. https://doi. org/10.1021/n1204196v

48 X. Li, W. Cai, J. An, S. Kim, J. Nah, D. Yang, R. Piner, A. Velamakanni, I. Jung, E. Tutuc, S. K. Banerjee, L. Colombo, and R. S. Ruoff: Science 324 (2009) 1312. https://doi.org/10.1126/science.1171245

49 S. Gilje, S. Han, M. Wang, K. L. Wang, and R. B. A. Kaner: Nano Lett. 7 (2007) 3394. https://doi.org/10.1021/ n10717715

50 X. Zhang, K. Li, H. Li, J. Lu, Q. Fu, and Y. Chu: Synth. Met. 193 (2014) 132. https://doi.org/10.1016/ j.synthmet.2014.04.007

51 N. Parveen, M. O. Ansari, S. A. Ansari, and M. H. Cho: J. Mater. Chem. A 4 (2016) 233. https://doi. org/10.1039/C5TA07963B

52 M. T. H. Aunkor, I. M. Mahbubul, R. Saidur, and H. S. C. Metselaar: RSC Adv. 6 (2016) 27807. https://doi. org/10.1039/C6RA03189G

53 J. Wang, E. C. Salihi, and L. Šiller: Mater. Sci. Eng. C 72 (2017) 1. https://doi.org/10.1016/j.msec.2016.11.017

54 Y. Shao, J. Wang, H. Wu, J. Liu, I. A. Aksay, and Y. Lin: Electroanalysis 22 (2010) 1027. https://doi. org/10.1002/elan.200900571

55 J. Chen, Y. Zhang, M. Zhang, B. Yao, Y. Li, L. Huang, C. Lia, and G. Shi: Chem. Sci. 7 (2016) 1874. https:// doi.org/10.1039/C5SC03828F

56 A. Ambrosi, C. K. Chua, A. Bonanni, and M. Pumera: Chem. Rev. 114 (2014) 7150. https://doi.org/10.1021/ cr500023c

57 S. Stankovich, D. Dikin, R. D. Piner, K. A. Kohlhaas, A. Kleinhammes, Y. Jia, Y. Wu, S. T. Nguyen, and R. S. Ruoff: Carbon 45 (2007) 1558. https://doi.org/10.1016/j.carbon.2007.02.034

58 W. S. Hummers and R. E. Offeman: J. Am. Chem. Soc. 80 (1958) 1339. https://doi.org/10.1021/ja01539a017

59 D. C. Marcano, D. V. Kosynkin, J. M. Berlin, A. Sinitskii, Z. Sun, A. Slesarev, L. B. Alemany, W. Lu, and J. M. Tour: ACS Nano 4 (2010) 4806. https://doi.org/10.1021/nn1006368

60 C. N. R. Rao, K. Sood, K. S. Subrahmanyam, and A. Govindaraj: Angew. Chem. Int. Ed. Engl. 48 (2009) 7752. https://doi.org/10.1002/anie.200901678

61 C. K. Chua and M. Pumera: Chem. Soc. Rev. 43 (2014) 291. https://doi.org/10.1039/c3cs60303b

62 R. B. A. Filho, C. M. B. de Araújo, A. M. S. Baptisttella, E. B. Batista, R. A. Barata, M. G. Ghislandi, and M. A. M. Sobrinho: Environ. Technol. (2019) https://doi.org/10.1080/09593330.2019.1581842

63 J. Chen, B. Yao, C. Li, and G. Shi: Carbon 64 (2013) 225. https://doi.org/10.1016/j.carbon.2013.07.055

64 M. Pumera: Chem. Soc. Rev. 39 (2010) 4146. https://doi.org/10.1039/C002690P

65 C. R. Minitha, V. S. Anithaa, V. Subramaniam, R. Thangavelu, and R. Kumar: ACS Omega 3 (2018) 4105. https://doi.org/10.1021/acsomega.7b02085

66 H. Nazemi, A. Joseph, J. Park, and A. Emadi: Sensors (Basel) 19 (2019) 1285. https://doi.org/10.3390/ s19061285

67 Y. Dan, Y. Lu, N. J. Kybert, Z. Luo, and A. T. C. Johnson: Nano Lett. 9 (2009) 1472. https://doi.org/10.1021/ n18033637

68 J. B. Sumner: J. Am. Chem. Soc. 45 (1923) 2378. https://doi.org/10.1021/ja01663a021

69 A. Manolis: Clin. Chem. 29 (1983) 5.

70 D. Zhang, J. Liu, C. Jiang, A. Liu, and B. Xia: Sens. Actuators B 240 (2017) 55. https://doi.org/10.1016/ J.SNB.2016.08.085

71 G. Gregis, S. Schaefer, J. B. Sanchez, V. Fierro, F. Berger, I. Bezverkhyy, G. Weber, J. P. Bellat, and A. Celzard: Mater. Chem. Phys. 192 (2017) 374. https://doi.org/10.1016/j.matchemphys.2017.02.015 
72 Z. Li, C. Xu, and J. Shu: Anal. Chim. Acta 964 (2017) 134. https://doi.org/10.1016/j.aca.2017.01.065

73 X. Huang, N. Hu, L. Zhang, L. Wei, H. Wei, and Y. Zhang: Synth. Met. 185-186 (2013) 25. https://doi. org/10.1016/J.SYNTHMET.2013.09.034

74 N. Hu, Z. Yang, Y. Wang, L. Zhang, and Y. Wang: Nanotechnology 25 (2014) 025502. https://doi. org/10.1088/0957-4484/25/2/025502

75 T. Le, V. Lakafosis, Z. Lin, C. P. Wong, and M. M. Tentzeris: Proc. Electronic Components and Technology Conference (IEEE, 2012) 1003. https://doi.org/10.1109/ECTC.2012.6248958

76 P. A. Pandey, N. R. Wilson, and J. A. Covington: Sens. Actuators B 183 (2013) 478. https://doi.org/10.1016/ j.snb.2013.03.089

77 R. Kumar, D. K. Avasthi, and A. Kaur: Sens. Actuators B 242 (2017) 461. https://doi.org/10.1016/j.snb.2016.11.018

78 F. Shen, D. Wang, R. Liu, X. Pei, T. Zhang, and J. Jin: Nanoscale 5 (2013) 537. https://doi.org/10.1039/ C2NR32752J

79 J. Hu, C. Zou, Y. Su, M. Li, N. Hu, H. Ni, Z. Yang, and Y. Zhang: J. Mater. Chem. C 5 (2017) 6862. https://doi. org/10.1039/c7tc01208j

80 S. Kumar, N. Kaur, K. Sharma, and A. Mahajan: RCS Adv. 7 (2017) 25229. https://doi.org/10.1039/c7ra02212c

81 K. Ganesan, S. Raza, and R. Vijayaraghavan: J. Pharm. Bioallied Sci. 2 (2010) 166. https://doi. org $/ 10.4103 / 0975-7406.68498$

82 B. C. Singer, A. T. Hodgson, H. Destaillats, T. Hotchi, K. L. Revzan, and R. G. Sextro: Environ. Sci. Technol. 39 (2005) 3203. https://doi.org/10.1021/ES049144U

83 S. L. Bartelt-Hunt, D. R. U. Knappe, and M. A. A. Barlaz: Crit. Rev. Environ. Sci. Technol. 38 (2008) 112. https://doi.org/10.1080/10643380701643650

84 M. Rowell, K. Kehe, F. Balszuweit, and H. Thiermann: Toxicology 263 (2009) 9. https://doi.org/10.1016/ j.tox.2009.05.015

85 J. B. Leikin, R. G. Thomas, F. G. Walter, R. Klein, and H. W. Meislin: Crit. Care Med. 30 (2002) 2346. https:// doi.org/10.1097/01.CCM.0000029196.42315.83

86 V. V. Singh, A. K. Nigam, S. S. Yadav, B. K. Tripathi, A. Srivastava, M. Boopathi, and B. Singh: Sens. Actuators B 188 (2013) 1218. https://doi.org/10.1016/j.snb.2013.08.013

87 N. Colozza, K. Kehe, T. Popp, D. Steinritz, D. Moscone, and F. Arduini: Environ. Sci. Pollut. Res. (Springer Link, 2018). https://doi.org/10.1007/s11356-018-2545-6

88 H. M. Hwang, E. Hwang, D. Kim, and H. Lee: Sci. Rep. 6 (2016) 33299. https://doi.org/10.1038/srep33299

89 A. Zurutuza and C. Marinelli: Nat. Nanotechnol. 9 (2014) 730. https://doi.org/10.1038/nnano.2014.225

90 M. S. Wiederoder, E. C. Nallon, M. W. Shannon, K. McGraw, V. P. Schnee, C. J. Bright, M. P. Polcha, R. Paffenroth, and J. R. Uzarski: ACS Sensors 2 (2017) 1669. https://doi.org/10.1021/acssensors.7b00550

91 A. Pappalardo, M. E. Amato, F. P. Ballistreri, V. la Paglia Fragola, G. A. Tomaselli, R. M. Toscano, and S. G. Trusso: J. Chem. Sci. 125 (2013) 869. https://doi.org/10.1007/s12039-013-0463-1

92 L. Bertilsson, K. Potje-Kamloth, H. Liess, I. Engquist, and B. Liedberg: J. Phys. Chem. B 102 (1998) 1260. https://doi.org/10.1021/JP973215C

93 G. S. Kulkarni, K. Reddy, W. Zang, K. Lee, X. Fan, and Z. Zhong: Nano Lett. 16 (2016) 695. https://doi. org/10.1021/acs.nanolett.5b04500

94 F. L. Migliorini, R. C. Sanfelice, L. A. Mercante, M. H. M. Facure, and D. S. Correa: Mater. Res. Express 7 (2020) 015601. https://doi.org/10.1088/2053-1591/ab5744

95 J. A. Hondred, J. Breger, N. Alves, S. A. Trammell, S. A. Walper, I. L. Medintz, and J. C. Claussen: ACS Appl. Mater. Interfaces 10 (2018) 11125. https://doi.org/10.1021/acsami.7b19763

96 G. Bolat and S. Abaci: Sensors (Basel) 18 (2018) 773. https://doi.org/10.3390/s18030773 